\title{
Low Complexity Link State Multipath Routing
}

\author{
Pascal Mérindol* \\ Université Catholique de Louvain (UCL) \\ Louvain la Neuve, Belgium \\ pascal.merindol@uclouvain.be
}

\author{
Jean-Jacques Pansiot and Stéphane Cateloin \\ LSIIT-CNRS, Université de Strasbourg (UdS) \\ Illkirch, France \\ \{pansiot,cateloin\}@unistra.fr
}

\begin{abstract}
Link state routing protocols such as OSPF or IS-IS currently use only best paths to forward IP packets throughout a domain. The optimality of subpaths ensures consistency of hop by hop forwarding although paths, calculated using Dijkstra's algorithm, are recursively composed. According to the link metric, the diversity of existing paths can be underestimated using only best paths. Hence, it reduces potential benefits of multipath applications such as load balancing and fast rerouting. In this paper, we propose a low time complexity multipath computation algorithm able to calculate at least two paths with a different first hop between all pairs of nodes in the network if such next hops exist. Using real and generated topologies, we evaluate and compare the complexity of our proposition with several techniques. Simulation results suggest that the path diversity achieved with our proposition is approximatively the same that the one obtained using consecutive Dijsktra computations, but with a lower time complexity.
\end{abstract}

\section{INTRODUCTION}

Routing is one of the key components of the Internet. Despite the potential benefits of multipath routing (e.g., [3] or [4]), most backbone networks still use unipath routing such as OSPF or IS-IS or their ECMP feature (Equal Cost MultiPath). With these routing protocols, the forwarding only changes upon topology variations and not upon traffic variations. Dynamic multipath routing (e.g., [13] or [12]) is able to provide several services such as load balancing, to reduce delays and improve throughput, and fast rerouting schemes in case of failures. The reliability of an IP network against failures and congestion depends on the reaction time necessary for the convergence of the underlying routing protocol. Proactive multiple paths calculation allows to accelerate this reaction time: pre-computed alternate paths can be

\footnotetext{
* Pascal Mérindol is partly funded by Trilogy, a research project (ICT-216372) supported by the European Community under its Seventh Framework Programme. The views expressed here are those of the author(s) only. The European Commission is not liable for any use that may be made of the information in this document.
}

directly used as backup paths without waiting for the routing protocol convergence. This proactive mechanism can improve the network response in case of troubles where such backup paths exist. To provide these functionalities, the set of forwarding alternatives has to be large enough to achieve a good path diversity. However, current routers only support ECMP. This feature corresponds to a simple variant of Dijkstra where equal cost paths are inherited along the shortest path tree (SPT). The optimality condition of sub-paths computed with ECMP restricts the number of loopfree paths and so reduces potential advantages of multipath routing.

Multipath routing protocols with hop by hop forwarding needs to validate a set of next hops such that the recursive composition between neighbor routers does not create forwarding loops (see [11], [12] and [14]). The first limitation is the complexity in time, space and the number of messages exchanged to compute and validate loopfree paths. In this paper, we propose a simple hop by hop scheme that does not require a signaling protocol to validate loopfree paths. If the validation procedure, whose goal is to verify the absence of loops, is local (without exchanging any message) and does not involve all routers, then the deployment can be incremental. Our approach is equivalent to ECMP in terms of time, space and message exchange complexity but allows to compute a greater diversity of forwarding alternatives.

In this paper, we propose the following contributions:

- two variants of the Dijkstra algorithm: DijkstraTransverse and multi-Dijkstra-Transverse.

- a proof that they compute at least two distinct next hops from the calculating node towards each node of the graph if such next hops exist.

- an evaluation of the efficiency and the complexity of our proposition compared to existing techniques.

\section{NOTATIONS AND CONTEXT}

Table I lists the graph definitions used in the paper. $G(N, E, w)$ denotes a directed graph $G$ with a set of 
nodes $N$, a set of edges $E$ and a strictly positive valuation of edges $w$. Notations are related to the multipath hop by hop forwarding context: computed paths are loopfree and first hop distinct. We order paths according to an additive metric $C$, and we focus on the best paths having distinct first hops. To distinguish equal cost paths, we consider the lexicographical order of first hops.

For simplicity reasons we do not consider the multigraph issue: a first hop is equivalent to a successor node, the next hop. The valuation $w$ denotes the weight of each directed link used by the routing protocol.

Let us define a safety property for multipath routing.

Definition: Loopfree routing property at the router level. A multipath routing protocol is loopfree if it always converges to a stable state such that when any router $s$ forwards a packet to any next hop $v$ towards any destination $d$, this packet never comes back to $s$.

With unipath or ECMP routing, the sub-path optimality condition guarantees the correctness of next hop composition. With hop by hop link state multipath routing using multiple unequal cost paths, two phases may be necessary to ensure loopfree routing: a path computation algorithm and a validation process. We do not consider validation processes using a signaling protocol (such as it can be done with distance vector routing messages). To increase the number of valid alternatives, the simplest rule to select a valid next hop $v$ on a router $s$ (such that $v \in \operatorname{succ}(s))$ is the downstream criteria which can be expressed as follows: $\quad C_{1}(v, d)<C_{1}(s, d)$

This rule is referenced in the IS-IS standard ISO 8473 , is used in OSPF-OMP [11] and is denoted LFI in [12] (with the particularity of avoiding routing loops in transient periods of topology changes). This rule is called one hop vision in [14] where Yang and Wetherall introduce a set of rules whose flexibility allows to increase the number of valid neighbors thanks to a two hops vision. This set of rules is more complex: the forwarding mechanism is specific to the incoming interface and allows forwarding loops at the router level but not at the link level. A packet is never forwarded twice through the same link but it can enter the same router twice.

In order to perform loopfree routing, the validation process needs to compute a set of candidate next hops. A candidate next hop is a first hop of a computed path which is not yet validated for loopfree routing. On a given calculating node (a root node $s$ ), the simplest way to obtain an exhaustive candidate set is to compute the SPT of all neighbor nodes. Thus, router $s$ can use the best costs information of its neighborhood. This approach is

\begin{tabular}{|c|c|}
\hline Notations & Definitions \\
\hline \hline$e=(e . x, e . y)$ & edge $e \in E$ connecting node $x$ to node $y$ \\
\hline$k^{-}(x), k^{+}(x)$ & incoming and outgoing degrees of node $x$ \\
\hline$s u c c(x)$ & set of neighbors of node $x\left(|\operatorname{succ}(x)|=k^{+}(x)\right)$ \\
\hline$P_{j}(s, d)=$ & $j^{t h}$ best loopfree path linking $s$ to $d$ \\
$\left(e_{1}, \ldots, e_{m}\right)$ & $\begin{array}{c}\text { This is the best path whose first edge is distinct } \\
\text { from the first edge of the } j-1 \text { first best paths }\end{array}$ \\
\hline$C_{j}(s, d)=$ & $\begin{array}{c}\text { cost of the path } P_{j}(s, d) \\
\sum_{i=1}^{m} w\left(e_{i}\right)\end{array}$ \\
\hline$N H_{j}(s, d)$ & $j^{t h}$ best next hop computed on $s$ towards $d$ \\
& This is the first hop $e_{1} . y$ of $P_{j}(s, d)$ \\
\hline
\end{tabular}

TABLE I: Notations

denoted $\mathbf{k D}$ in the following, and our analysis uses this technique as a reference. The complexity of $\mathrm{kD}$ depends on the number of neighbors: $k^{+}(s)+1$ instances of the Dijkstra algorithm are necessary to compute the local and neighborhood best costs. If a router has a large number of interfaces, the computation time can be too long. Even if this calculation is typically done offline, when a congestion or a failure occurs during this period, the router is unable to perform the traffic switching. Another way is to use an enhanced SPT algorithm to locally compute multiple paths for each destination. For example, algorithms and implementations presented in [9] are designed to compute the set of $K$-shortest loopfree paths, but do not guarantee that these paths are first hop distinct. The $K$-shortest loopfree paths problem is not suited for simple hop by hop forwarding. Indeed, in order to forward packets via these $K$ explicit paths, a signaling protocol is necessary to mark routes from the ingress router towards each egress router. Here we focus on distinct first hops computation $\left(K \leq k^{+}(s)\right)$, and paths are implicity stored as candidate next hops. The objective of our approach is to compute a set of loopfree first hop disjoint paths with a lower complexity than kD. For this purpose, we calculate a set of costs $\left\{C_{j}(s, d)\right\}_{\forall d \in N}$ containing at least two entries for each destination node $d$ in the graph. With an enhanced SPT algorithm able to compute such a set, rule (1) becomes:

$$
C_{j}(s, d)-w(s, v)<C_{1}(s, d)
$$

If $v=N H_{j}(s, d)$ satisfies rule (2), then $(s, v)$ is a valid next hop. Thus, the $j^{t h}$ next hop $v$ can be used by $s$ to reach $d$ : it satisfies the loopfree routing property at the router level. Note that $\forall d \in N, C_{j}(s, d)-w(s, v) \geq$ $C_{1}(v, d)$. Our approach follows these three steps:

1) it uses an unmodified link state routing protocol to obtain topological advertisements,

2) it uses a multipath computation algorithm instead of Dijkstra to compute candidate next hops,

3 ) it uses condition (2) to select valid next hops. 


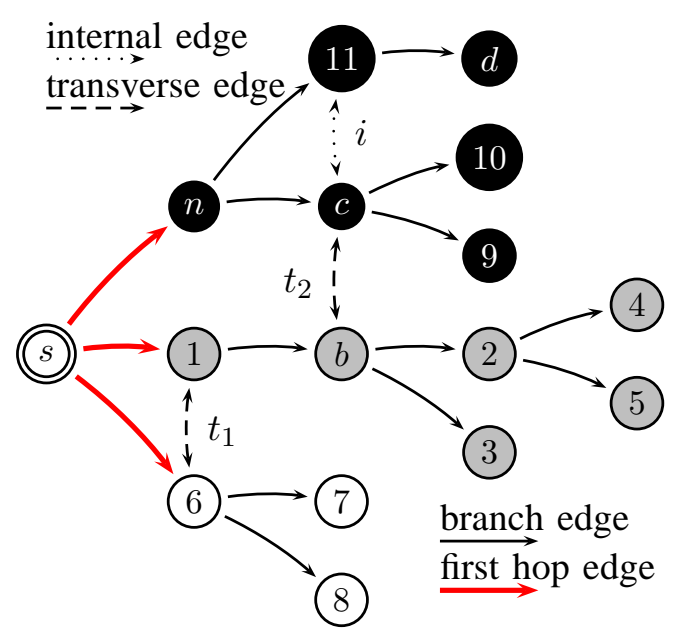

Fig. 1: Edge partition example

\section{CANDIDATE NEXT HOPS COMPUTATION}

This section describes our path computation algorithms and an original edge partition analysis. Given a root node $s$, the set of edges of a graph can be partitioned into four subsets (we consider edges in both directions):

- Edges corresponding to first hops of primary paths.

- Edges belonging to the branches.

- Transverse edges connecting two distinct branches or connecting the root $s$ and a branch without being the first hop of a primary path.

- Internal edges linking nodes of the same branch without belonging to this branch.

These four subsets exhaustively describe $E$ because the set of branches contains all nodes (except the root node $s$ ) in the graph. Fig. 1 illustrates an edge partition on a simple graph. In this graph (we consider $w$ as a constant function), there are three branches (black, gray and white nodes), two transverse edges (dashed arcs denoted $t_{1}$ and $t_{2}$ ) and one internal edge (dotted arc denoted $i$ ). Edges $(s, n),(s, 1)$ and $(s, 6)$ correspond to the three first hops (red arcs) linking $s$ to the three branches.

With multipath hop by hop routing, the primary path denotes the optimal path depending on a given metric and a lexicographic order to rank equal cost paths. Thus, for a given pair $(s, d)$, an alternate path is a path whose first edge is distinct from the first one of the primary path $P_{1}(s, d)$. More generally, if the forwarding mechanism is distributed such as with hop by hop routing, then all alternate paths are first hop distinct.

Our algorithms compute transverse paths (see Table II for related definitions). The path $(s, 1, b, c)$ is simple transverse and the path $(s, 1, b, c, n)$ is backward transverse. Paths $\mathcal{P}=(s, 1, b, c, n, 11, d)$ and $\mathcal{P}^{\prime}=(s, 6,1, b)$ are both forward transverse. However, $\mathcal{P}$ contains a sub path $(s, 1, b, c, n) \in \operatorname{Pbt}(s, n)$ whereas $\mathcal{P}^{\prime}$ contains a

\begin{tabular}{|c|c|}
\hline Terms & Definitions \\
\hline \hline branch $_{h}(s)$ & subtree of the SPT rooted at a neighbor $h$ of $s$ \\
\hline transverse edge & an edge is transverse if it connects \\
& two distinct branches branch $_{h}(s)$ and \\
& branch $_{h^{\prime}}(s)$ or if it connects the root $s$ \\
& and a node $n \neq h$ in a branch ${ }_{h}(s)$
\end{tabular}

TABLE II: Multipath terminology

sub path $(s, 6,1) \in \operatorname{Pt}(s, 1)$. The routing information base cannot directly use the set of candidate next hops corresponding to the first hops of 1-transverse path to perform forwarding, since routing loops may occur. Our approach needs a validation mechanism to select valid next hops among candidate next hops in order to guarantee the safety of forwarding. For that purpose, we consider the downstream criteria, the rule (2).

\section{A. DT and mDT algorithms}

In [7], we have proposed and described the DijkstraTransverse algorithm (DT). Here, we focus on DT properties that we have not presented in [7] (see section III-B) and on a DT improvement that we call multi-DT (mDT). However, the basics of DT and mDT are similar. To sum up, DT and mDT compute a multipath cost matrix on a given root node (denoted $s$ in the following). A multipath cost matrix $M c$ contains an overestimation of best costs for all $(|N|-1)$ destinations and via all possible $\left(k^{+}(s)\right)$ neighbors of $s$. The goal of our algorithms is to calculate a set of candidate next hops associated to path costs via each neighbor.

The calculation consists in two main stages:

1- Compute the best path tree and transverse edges.

2- Compute backward and forward transverse paths.

At each iteration, our algorithms compute the best 1transverse paths depending on the first hop. Without an optimized structure to implement the best costs vector (the priority queue, denoted $T c$ ), the complexity of DT for each calculating node $s$ is in the worst case:

$$
O\left(|N|^{2}+|E|+|N| \times k^{+}(s)\right)=O\left(|N|^{2}\right)
$$

DT adds a time complexity proportional to the outgoing 
degree of the given root node $s$ compared to Dijkstra. With a Fibonacci heap [5] to implement $T c$, it is possible to reduce the time complexity to:

$$
O\left(|N| \log _{2}|N|+|E|+|N| \times k^{+}(s)\right)
$$

The minimum extraction has an unitary cost whereas the minimum suppression has an amortized cost in $O\left(\log _{2}(|N|)\right)$. For simplicity reasons, evaluations results presented in this paper only rely on array lists.

The set of candidate next hops computed with DT does not always include all next hops corresponding to equal best cost paths. mDT (the complete algorithm is described in the techreport [8]) is able to solve this problem. With mDT, only the first computation phase of DT is modified by using a next hop matrix denoted $T p$. This matrix represents the existence of a next hop per neighbor for each destination. $T p$ is updated at each edge exploration. Candidate next hops recording follows a transitive rule: $T p(k, y) \leftarrow T p(k, x)$ with $y \in \operatorname{succ}(x), k \in \operatorname{succ}(s)$. Initially, if $x=s$ then $T p(y, y) \leftarrow y$. With ECMP, the update of $T p$ is performed only if $T c(x)+w(x, y) \leq T c(y)$. We have chosen to generalize this approach to improve the upper bound on the cost of forward transverse paths composed with a backward transverse path. This generalization increases the number of validated next hops. Indeed, during the exploration of the set of successors of node $x$, if node $y$ is not already marked, it inherits all forwarding alternatives of $x$, including when $(x, y)$ is an internal edge. In this case, the next hop inheritance is not restricted to branches as with DT: $y$ is not the son of $x$ on a primary path. mDT allows to use all forwarding alternatives already computed towards $x$. This set of paths is not limited to 1-transverse alternatives, it can contain alternate paths with several internal or transverse edges. The mDT computation is based on the order of node exploration which depends on the rank of costs stored in $T c$. With $\mathrm{mDT}$, the first computation phase is able to calculate all candidate next hops corresponding to ECMP alternatives. Recursively, the cost inheritance takes into account all the sets of equal best cost paths for all marked nodes. The complexity of $\mathrm{mDT}$ is slightly greater than the one of DT: for each iteration of the main loop, $k^{+}(s)$ operations are necessary to execute the inheritance of next hops and their costs. The worst case complexity of mDT is in $O\left(|N|^{2}+E \times k^{+}(s)\right)$ without an optimized structure for $T c$.

\section{B. Properties of $D T$ and $m D T$}

Our algorithms are able to compute at least one alternate path towards each destination if such alternative exists. Proofs are given in the mDT techreport [8].

Property 1. DT computes all 1-transverse paths, and mDT computes all paths computed with DT and all equal best cost paths.

The proof of these properties relies on next hops inheritance performed by DT and mDT (see [7]).

Now, let us define a major property of 1-transverse paths. The demonstration of this property relies on two lemmas.

Property 2. If there exists an alternate path $P(s, d)$, then there exists a 1-transverse path between $s$ and $d$.

Lemma 1. If there exists an alternate path $\mathcal{P}$ from $s$ to $d$, then there exists a path from $s$ to $d$ whose cost is not greater than the one of $\mathcal{P}$ and containing only one transverse edge.

Figure 1 illustrates lemma 1 . Let $\circ$ be the operator representing the path concatenation. The 2-transverse path $\mathcal{P}=(s, 6,1, b, c)$ between $s$ and $c$ via the neighbor node 6 uses $\operatorname{branch}_{1}(s)$ to reach the transverse edge $(b, c)$. There exists an alternate simple transverse path $\mathcal{P}^{\prime}=P_{1}(s, b) \circ((b, c))$. Note that the existence of an alternate path $\mathcal{P}$ with several transverse edges implies that DT and mDT implicitly record a 1-transverse path $\mathcal{P}^{\prime}$ in the cost matrix $M c$ with a cost lower or equal to the cost of $\mathcal{P}$.

Lemma 2. If there exists an alternate path from $s$ to $d$ with one transverse edge, then there exists a 1-transverse path linking $s$ and $d$.

Figure 1 illustrates lemma 2. Although the alternate path $(s, 1, b, c, 11, d)$ is not 1-transverse because it contains an internal edge $i=(c, 11)$, there exists a forward transverse path $(s, 1, b, c, n, 11, d)$. In this case, the internal edge $i$ is bypassed with a backward composition followed by a forward composition. It allows to compute the alternate next hop 1 to reach $d$.

Thanks to the backward and forward composition, if there exists a 1-transverse path, then DT finds it. These two phases allow to use edges of the SPT in both directions. Moreover, DT considers all transverse edges because, as it is the case for the classical Dijkstra algorithm, all edges must be explored in order to mark all nodes. The difference is that DT implicitly stores longer or equal cost paths in the cost matrix.

\section{Evaluation}

We have used the Network Simulator 2 (ns2, [2]) to compare several routing approaches. We have extended 


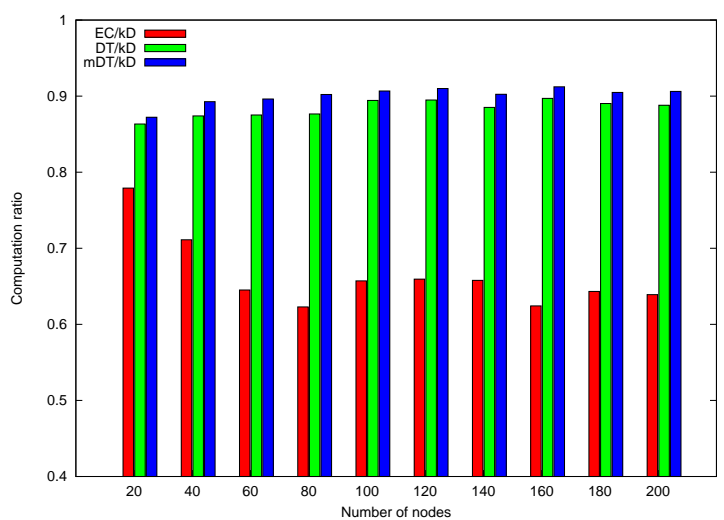

Fig. 2: Number of candidate next hops (Igen topologies)

(see [1]) the link state routing module of ns2 to support $\mathrm{DT}, \mathrm{mDT}, \mathrm{kD}$ and the downstream criteria.

\section{A. Topologies and simulations setup}

We present results obtained on three different kinds of topologies. The first category of networks are real topologies with actual IGP weights (for confidentiality, we approximate their size in Table III). Topologies denoted ISP1 and ISP2 are commercial networks covering an European country. ISP3 and ISP4 are Tier-1 ISP networks. The second category of networks were chosen among the Rocketfuel inferred set of maps (see [6]).

We have also used the Igen topology generator ([10]) to generate 10 topologies containing between 20 and 200 nodes using the K-medoid parameter, the delaytriangulation heuristic and a 2 -sprint pop design. The $\mathrm{K}$ parameter that determine the number of routers per cluster is chosen such that $\mathrm{K}=\frac{|N|}{10}$. Each cluster contains approximatively 10 routers for each generated topology. These parameters offer a great physical diversity to measure the relevance of our proposition to achieve the same level of diversity as computed with $k D$. The link valuation used for this third category is the inverse of the link capacity. The mean degree, denoted $k$, is approximatively the same for each generated topology: $k \sim 4$. These networks represent access backbones and contain two kinds of links: $155 \mathrm{Mbps}$ for access links and 10Gbps for backbone links.

\section{B. Results}

First, we have measured the path diversity (see Fig.2). We have calculated the total number of candidate next hops obtained with ECMP (denoted EC), DT, mDT, and multiple Dijkstra computations $(\mathrm{kD})$. Results are represented as a performance ratio between the considered technique and $\mathrm{kD}$ for all routers of a given network. $\mathrm{kD}$ provides the best diversity but with a higher computation

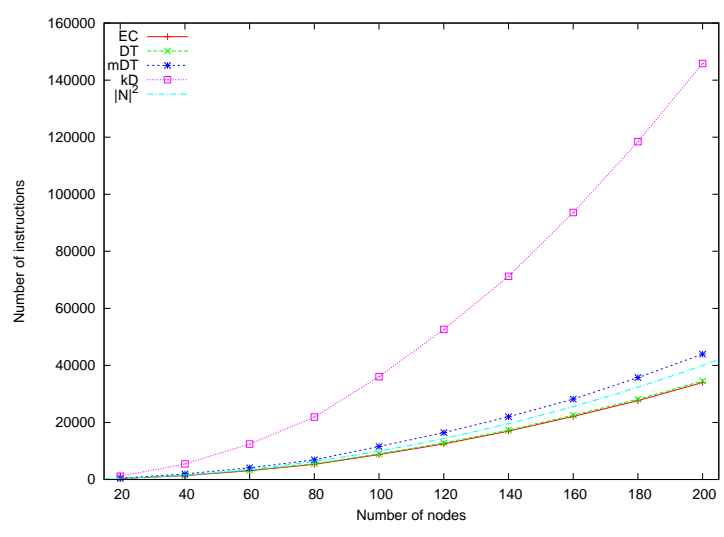

Fig. 3: Number of operations (Igen topologies)

cost. We observe that DT and mDT are able to compute approximatively $90 \%$ of candidate next hops obtained with $\mathrm{kD}$, while ECMP obtains a performance ratio only between $60 \%$ and $80 \%$.

Then, we have compared the time complexities of the fore mentionned algorithms (see Fig. 3). We have represented the execution time measured in number of operations needed by $\mathrm{DT}, \mathrm{mDT}$ and $\mathrm{kD}$ to compute their set of candidate next hops. The number of operations is an average computed for each router. This value takes into account all operations necessary to extract the min of $T c$ and perform update of $T c, M c$ and $T p$. We notice that the time saved with DT or mDT is really significant compared to $\mathrm{kD}$. The number of operations needed by $\mathrm{kD}$ is approximatively $k \times|N|^{2}$ whereas mDT and DT need approximatively $|N|^{2}$ operations. This complexity is equivalent to the worst case of an ECMP computation. The time complexity upper bound is reached because some routers have a high degree of connectivity.

Finally, we have compared the number of validated next hops that are selected with the downstream criteria (rule 2) depending on the computation algorithm (see Fig. 4). We remark that mDT allows to validate as many next hops as $\mathrm{kD}$. This result can be explained by the specific valuation of our set of generated topologies: there are only two very different weights used in these networks. Results given in Table III illustrate the same evaluation of performance ratios and complexity on the set of real and inferred topologies. For these sets of topologies, Table III also shows candidate and valid next hops average per destination obtained with $\mathrm{kD}$. Diversity ratio results are similar to the ones obtained with Igen although degrees and weights distributions are completely different. The main difference comes from the time complexity evaluation. On these topologies, the maximum degree of nodes is two times lower than 


\begin{tabular}{|c|c|c|c|c|c|c|c|c|c|c|c|c|c|c|}
\hline \multirow{3}{*}{$\begin{array}{c}\text { Network } \\
\text { name }\end{array}$} & \multirow{2}{*}{\multicolumn{2}{|c|}{ Size }} & \multicolumn{4}{|c|}{ Candidate next hops } & \multicolumn{4}{|c|}{ Validated next hops } & \multicolumn{4}{|c|}{ Number of operations } \\
\hline & & & \multirow{2}{*}{$\frac{\text { mean }}{\mathrm{kD}}$} & \multicolumn{3}{|c|}{ ratio/kD (\%) } & \multirow{2}{*}{$\frac{\text { mean }}{\mathrm{kD}}$} & \multicolumn{3}{|c|}{ ratio/kD (\%) } & \multirow{2}{*}{$\frac{\text { mean }}{\mathrm{kD}}$} & \multicolumn{3}{|c|}{ ratio/kD (\%) } \\
\hline & $|N|$ & $E$ & & $\mathrm{EC}$ & DT & mDT & & $\mathrm{EC}$ & DT & mDT & & $\mathrm{EC}$ & DT & mDT \\
\hline ISP1 & $\overline{25}$ & 50 & 1.46 & $\overline{76}$ & 97 & 97 & 1.10 & 97 & 100 & 100 & 489 & 60 & 66 & 75 \\
\hline$\overline{\text { ISP2 }}$ & $\overline{50}$ & 200 & 3.58 & $\overline{43}$ & 93 & 97 & 1.79 & 69 & 89 & $\overline{94}$ & 6730 & 30 & 32 & 32.5 \\
\hline ISP3 & 110 & 350 & 2.70 & 55 & 89 & 92 & 1.45 & 82 & 97 & 99 & 8079 & 38 & 41 & 43.5 \\
\hline $\begin{array}{ll}\text { ISP4 } \\
\end{array}$ & 210 & 880 & 3.73 & 44 & 86 & 88 & 1.81 & $\overline{72}$ & 96 & 99 & 41747 & 27 & 28 & 31 \\
\hline 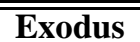 & $\overline{\overline{79}}$ & $\overline{\overline{294}}$ & $\overline{\overline{3.58}}$ & $\overline{\overline{44}}$ & 88 & 96 & 1.73 & 58 & $\overline{994}$ & 99 & 5569 & 29 & $\overline{34}$ & 37 \\
\hline Ebone & 87 & 322 & 3.49 & 46 & 90 & 96 & 1.76 & 77 & 93 & 99 & 9698 & 30 & 33 & 36 \\
\hline Telstra & 104 & 304 & 2.30 & 72 & 92 & 95 & 1.30 & 90 & 98 & 99 & 6526 & 54 & 57 & 59 \\
\hline Above & 141 & 748 & 5.29 & 34 & 86 & 97 & 2.50 & 58 & 89 & 99 & 40143 & 18.5 & 20 & 23 \\
\hline Tiscali & 161 & 656 & 3.68 & 54 & 91 & 97 & 1.97 & 74 & 92 & 97 & 31044 & 27 & 29 & 32 \\
\hline
\end{tabular}

TABLE III: Evaluation results on real and inferred topologies

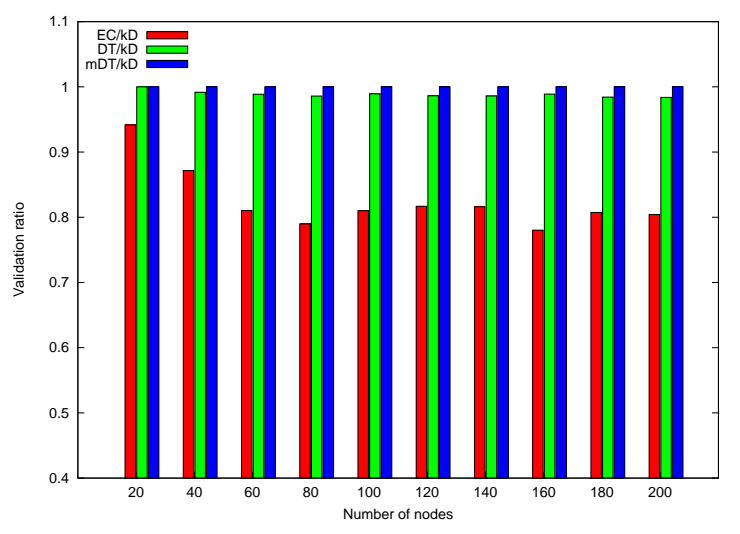

Fig. 4: Number of validated next hops (Igen topologies)

with Igen topologies. The measured complexity is far away from the theoretical worst case. More generally, parameters such as the valuation function $w$ or the degree distribution may strongly influence complexity or performance measures. For example, if $w$ is a constant function, rule (2) is equivalent to ECMP. Thus, in this case, the number of valid next hops is the same for $\mathrm{mDT}, \mathrm{kD}$ and ECMP. Another key point is the fact that the alternate paths which are not computed with mDT have a cost generally much greater than the one of the primary path. That is why the ratio of loopfree alternatives between $\mathrm{mDT}$ and $\mathrm{kD}$ is close to $100 \%$.

\section{CONCLusion}

Multipath routing enhances the network reachability and allows load balancing to circumvent congestions or failures. However, the overhead imposed by signaling messages, the time and space complexity can hamper its deployment. In this paper, we propose a simple scheme that is able to generate a greater path diversity than ECMP with an equivalent overhead. Our path computation algorithms, Dijkstra-Transverse, and its improvement multi-DT, allow to compute at least two candidate next hops between all pairs of routers if such next hops exist. To validate candidate next hops in a distributed manner, we have considered the simplest loopfree routing rule, the downstream criteria. Our evaluations suggest that the gain of time is very significant. We show that the number of next hops validated with the downstream criteria is slightly the same using mDT or a Dijkstra computation per neighbor. Moreover, our proposition can be incrementally integrated in OSPF or IS-IS by replacing the path computation algorithm without any change in the protocol.

\section{REFERENCES}

[1] "Implementation of dt and mdt in ns2," http://www-r2. u-strasbg.fr/ $\sim$ merindol/uploads/Research/DT.tar.gz.

[2] "The network simulator- ns2," http://www.isi.edu/nsnam/ns.

[3] R. Banner and A. Orda, "Multipath routing algorithms for congestion minimization," IEEE/ACM Trans. Netw., 2007.

[4] I. Cidon, R. Rom, and Y. Shavitt, "Analysis of multi-path routing," IEEE/ACM Trans. Netw., vol. 7, no. 6, 1999.

[5] T. H. Cormen, C. E. Leiserson, R. L. Rivest, and C. Stein, Introduction to Algorithms, 2nd ed. The MIT Press, 2001.

[6] R. Mahajan, N. Spring, D. Wetherall, and T. Anderson, "Inferring link weights using end-to-end measurements," in $A C M$ SIGCOMM Internet Measurement Workshop, 2002.

[7] P. Mérindol, J.-J. Pansiot, and S. Cateloin, "Path computation for incoming interface multipath routing," in ECUMN, 2007.

[8] P. Merindol, J.-J. Pansiot, and S. Cateloin, "The mdt algorithm," LSIIT-UdS, arXiv:0904.0217v1 RR-PM01-09, 2009.

[9] M. Pascoal, "Implementations and empirical comparison for k shortest loopless path algorithms," in The Ninth DIMACS Implementation Challenge: The Shortest Path Problem, 2006.

[10] B. Quoitin, "Topology generation through network design heuristics," http://www.info.ucl.ac.be/ bqu/igen/.

[11] C. Villamizar, "Ospf optimized multipath (ospf-omp): draft-ietfospf-omp-02.txt," IETF, Draft, Feb. 1999.

[12] S. Vutukury, "Multipath routing mechanisms for traffic engineering and quality of service in the internet," Ph.D. dissertation, 2001.

[13] H. Wang and al., "Cope: traffic engineering in dynamic networks," in SIGCOMM, 2006.

[14] X. Yang and D. Wetherall, "Source selectable path diversity via routing deflections," in SIGCOMM, vol. 36, 2006. 